\title{
Life Satisfaction Set Point: Stability and Change
}

\author{
Frank Fujita \\ Indiana University South Bend
}

\author{
Ed Diener \\ University of Illinois at Urbana-Champaign \\ and the Gallup Organization
}

\begin{abstract}
Using data from 17 years of a large and nationally representative panel study from Germany, the authors examined whether there is a set point for life satisfaction (LS) — stability across time, even though it can be perturbed for short periods by life events. The authors found that $24 \%$ of respondents changed significantly in LS from the first 5 years to the last 5 years and that stability declined as the period between measurements increased. Average LS in the first 5 years correlated .51 with the 5 -year average of LS during the last 5 years. Height, weight, body mass index, systolic and diastolic blood pressure, and personality traits were all more stable than LS, whereas income was about as stable as LS. Almost $9 \%$ of the sample changed an average of 3 or more points on a 10-point scale from the first 5 to last 5 years of the study.
\end{abstract}

The purpose of this study was to explore the degree to which life satisfaction (LS) varies around an individual set point, a personal baseline that remains constant over time. The set-point concept was borrowed from the idea of a weight set point and implies that there is a stable baseline of LS, with homeostatic forces returning it to its original level after life events or changing circumstances change it. We analyzed in a large probability sample whether annual reports of LS followed a set-point pattern over a 17-year period.

The concept of set point is a pivotal one to the field of subjective well-being (SWB) for both theoretical and applied reasons. In terms of theory, the idea of set point makes strong predictions about the relation of temperament and events in influencing SWB. In terms of practical importance, interventions to change society or to help individuals must be considered in a different light if they cannot hope to improve people's SWB. Thus, the question of whether a personal set point exists is of immense importance to the field of psychology.

In recent years, a large literature has been focused on the understanding of SWB (Kahneman, Diener, \& Schwarz, 1999; Myers, 1992). Headey and Wearing (1992) proposed that SWB has a baseline for each individual and that it returns to this set point after unusual events perturb it away from the stable level that is

Frank Fujita, Department of Psychology, Indiana University South Bend; Ed Diener, Psychology Department, University of Illinois at Urbana-Champaign, and the Gallup Organization, Princeton, New Jersey.

The Framingham Heart Study was conducted and supported by the National Heart, Lung, and Blood Institute (NHLBI) in collaboration with Boston University. This article was not prepared in collaboration with investigators of the Framingham Heart Study and does not necessarily reflect the opinions or views of the Framingham Heart Study, Boston University, or the NHLBI. We thank Daniel Kahneman for comments on this research project.

Correspondence concerning this article should be addressed to Ed Diener, Psychology Department, University of Illinois at Urbana-Champaign, 603 East Daniel Street, Champaign, IL 61820. E-mail: ediener@ s.psych.uiuc.edu determined by the recurring life events that result from individuals' personality predispositions. Similarly, Lykken and Tellegen (1996) proposed that long-term SWB is determined primarily by a person's genetically based dispositions, although they maintained that events can temporarily move individuals above or below their baselines. The set-point approach to SWB derives in part from the dramatic finding that people over time often adapt to bad and good conditions, an idea first advanced by Brickman and his colleagues (Brickman \& Campbell, 1971; Brickman, Coates, \& JanoffBulman, 1978) and labeled "the hedonic treadmill." Thus, people might initially react strongly to events but then return to a baseline of LS that is determined by their inborn temperaments.

Several lines of data suggest that a set-point model to some degree describes LS over time. First, moderate to substantial stability coefficients have been uncovered in SWB. For example, Eid and Diener (2004) found substantial stability in LS over a period of 4 weeks, with $74 \%$ of the reliable variance being due to stable individual differences, $16 \%$ of the variance due to occasionspecific influences, and the remaining variance due to random error. Second, the impressive heritability research of the Minnesota Twin Study group indicates that individual differences in SWB have a strong genetic component (Tellegen et al., 1988) and that long-term stable levels of SWB might be predominantly genetic in origin.

Despite evidence for stability and heritability, there are also indications that LS is influenced by events, and there is suggestive evidence that even long-term levels can be affected by circumstances. There are nation-level differences in LS that persist over time as well as long-term mean shifts in average LS when living conditions in a society change (Inglehart \& Klingemann, 2000). Also, it has been found that even after a period of several years, people do not seem to fully adapt to certain life events such as widowhood (Lucas, Clark, Georgellis, \& Diener, 2003) and unemployment (Lucas, Clark, Georgellis, \& Diener, 2004). Further, Schimmack, Diener, and Oishi (2002) found that LS shifts when relevant information used by the respondent changes. Thus, there is a persistent belief that despite the impact of personality on SWB, circumstances can matter, even in the long run. In his recent book 
on happiness, Lykken (1999), a core member of the Minnesota group, suggested ways that a person might change his or her set point.

Although previous research has suggested that LS is stable but can change somewhat from occasion to occasion, it has not yet fully explored the set-point idea. For one thing, most past research is cross-sectional, or based on only a few points in time. In a short period of time, such as a few years, a person might be pushed from her or his baseline by intense events and then later return to the set point. Furthermore, momentary situational factors such as mood and the priming of particular information can substantially influence LS on specific testing occasions (Schwarz \& Strack, 1999) and yet not influence average LS when it is sampled over long periods of time. Therefore, it is difficult without repeated measures over a long period of time to disentangle short-term shifts from long-term changes and to estimate the degree of long-term stability that might be hidden by momentary influences. Another limitation of past research is that it is often conducted on college students or convenience samples, and therefore the stability of SWB in the general population is unknown.

We used data from the German Socio-Economic Panel (GSOEP), a nationally representative longitudinal annual panel study of Germans. By using a nationally representative sample, we overcome one of the limitations of past research that often used college students or other convenience samples. By using an annual panel data collection design, we were able to disentangle momentary situational factors, which show up in our study as error of measurement, from long-term changes that show stability in relation to additional waves of data. Thus, this study allows much stronger inferences than previous research that used a convenience sample or a design with few measurement points in time.

We hypothesized that despite momentary influences on the measures, there would be substantial stability in LS (because of personality and also because of the stability of many life circumstances). Even though people are influenced by momentary factors in their LS judgments, they are likely to access some of the same information about their lives when making LS judgments on two occasions, and some of this information is likely to be stable. However, on the basis of past findings, we also predicted that some people would show significant long-term changes that went beyond single occasions of measurement. In other words, we predicted that the baseline could change. Just as some people show long-term weight gain or loss, even in the face of homeostatic influences on body mass, we hypothesized that some individuals would change in their long-term levels of LS because of strong alterations in life circumstances such as widowhood and unemployment.

In addition to exploring the stability of LS over years, we examined some related questions. First, we analyzed whether stability was greater for satisfied or dissatisfied individuals. Perhaps the average person in the satisfied range is stable, but some less satisfied individuals are less stable because they are more reactive to events (see Eid \& Diener, 1999). A second ancillary question we addressed is how LS compares in stability with height, weight, the body mass index, income, personality, and physiological measures like systolic and diastolic blood pressure.
Method

\section{Sample}

The GSOEP is a nationally representative longitudinal annual panel study of Germans. It was described by Haisken-De New and Frick (1998). The 3,608 participants, selected only if they completed the LS question (see below) each year from 1984 to 2000, included 1,709 male and 1,899 female respondents. The year of birth of the participants ranged from 1902 to 1968 , with a mean of $1944.8(S D=13.8)$.

\section{Measures}

Data are reported on the subsample that responded to the question, "How happy are you at present with your life as a whole?" on a 0 (totally unhappy) to 10 (totally happy) scale each year from 1984 to 2000 . We call the answer to this question "life satisfaction" (LS) because it refers to a reflection about life as a whole rather than the experience of pleasant emotions. In addition, self-reported total monthly household income in deutsch marks was collected annually.

\section{Results}

Because the purpose of our study was to determine the accuracy of a set-point model of LS, the individual participants were sorted by the within-person standard deviations of their LS across the 17 years. Table 1 presents raw data for 5 people the 25 th, 50th, and 75th percentiles for the variability of LS. These individual scores give the reader a feeling for how much people changed on LS from year to year and a sense of more variable and less variable individuals. The mean within-person standard deviation was 1.28; within-person standard deviations for the 25th, 50th, and 75th percentiles were $0.90,1.22$, and 1.59 , respectively. It is noteworthy that the average between-person standard deviation of LS was 1.78 , indicating that there is more variability between individuals than there is within the average individual. As can be seen in Table 1, many individuals changed substantially from year to year. The most stable group shown varies in a tight range, moving up or down a point from their average level. The less stable group shows large swings, and it appears that in several cases their baseline moved. ${ }^{1}$

Year-to-year changes do not give a complete picture of the set-point idea because there might be short-term fluctuations due to recent life events but long-term stability. Thus, we examined the idea of set point in terms of a longer baseline period. We defined a baseline as the average LS judgment of 5 consecutive years. We next examined the beginning baseline (1984-1988) and compared it with the ending baseline (1996-2000). We would expect, under the stable baseline hypothesis, that $5 \%$ of the participants would have a significant change of baseline using a dependent $t$ test with an alpha of .05 . Of the 3,608 participants, 852 (24\%) had a significant change of baseline, with $219(6 \%)$ experiencing a rising baseline and $633(18 \%)$ experiencing a falling baseline. Thus, the number of participants experiencing a significant change of baseline was more than four times what would be expected under the

\footnotetext{
${ }^{1}$ To further examine the mean- and within-person standard deviation of LS, an exploratory regression analysis was performed using the sex and year of birth of the participants. The regression analysis indicated no main effects of either year of birth or sex that were significant predictors of either mean- or within-person standard deviation of LS.
} 
Table 1

Raw Data for 5 Respondents at the 25th, 50th, and 75th Percentiles of Variability (Standard Deviation) Across 17 Years of Life Satisfaction Judgments

\begin{tabular}{|c|c|c|c|c|c|c|c|c|c|c|c|c|c|c|c|c|c|}
\hline \multirow[b]{2}{*}{ Respondent } & \multicolumn{17}{|c|}{ Year } \\
\hline & 1984 & 1985 & 1986 & 1987 & 1988 & 1989 & 1990 & 1991 & 1992 & 1993 & 1994 & 1995 & 1996 & 1997 & 1998 & 1999 & 2000 \\
\hline \multicolumn{18}{|c|}{25 th percentile } \\
\hline 1 & 9 & 7 & 6 & 5 & 7 & 6 & 6 & 7 & 7 & 6 & 8 & 7 & 7 & 7 & 7 & 7 & 6 \\
\hline 2 & 5 & 3 & 4 & 5 & 5 & 5 & 5 & 5 & 5 & 6 & 7 & 5 & 5 & 4 & 6 & 5 & 4 \\
\hline 3 & 6 & 4 & 6 & 5 & 6 & 6 & 8 & 6 & 7 & 7 & 6 & 6 & 7 & 6 & 7 & 7 & 6 \\
\hline 4 & 9 & 9 & 8 & 8 & 7 & 8 & 10 & 10 & 10 & 9 & 10 & 9 & 9 & 9 & 8 & 8 & 8 \\
\hline 5 & 8 & 10 & 8 & 8 & 7 & 6 & 8 & 9 & 7 & 9 & 8 & 9 & 8 & 8 & 8 & 8 & 8 \\
\hline \multicolumn{18}{|c|}{ 50th percentile } \\
\hline 1 & 5 & 4 & 3 & 2 & 1 & 4 & 3 & 4 & 5 & 4 & 4 & 4 & 5 & 3 & 5 & 4 & 6 \\
\hline 2 & 8 & 10 & 10 & 7 & 10 & 9 & 7 & 9 & 6 & 7 & 9 & 7 & 9 & 8 & 8 & 9 & 9 \\
\hline 3 & 7 & 8 & 6 & 7 & 6 & 5 & 5 & 4 & 5 & 4 & 5 & 6 & 5 & 6 & 6 & 8 & 7 \\
\hline 4 & 8 & 6 & 7 & 3 & 8 & 8 & 8 & 7 & 7 & 6 & 7 & 7 & 7 & 6 & 7 & 8 & 7 \\
\hline 5 & 7 & 8 & 5 & 5 & 6 & 8 & 7 & 7 & 6 & 8 & 6 & 8 & 4 & 6 & 6 & 5 & 6 \\
\hline \multicolumn{18}{|c|}{ 75th percentile } \\
\hline 1 & 4 & 4 & 4 & 5 & 7 & 6 & 8 & 8 & 5 & 8 & 8 & 8 & 8 & 7 & 8 & 6 & 7 \\
\hline 2 & 10 & 8 & 8 & 7 & 5 & 6 & 7 & 3 & 7 & 6 & 7 & 5 & 5 & 7 & 5 & 7 & 7 \\
\hline 3 & 4 & 9 & 7 & 9 & 9 & 5 & 7 & 6 & 6 & 5 & 6 & 6 & 6 & 4 & 6 & 5 & 5 \\
\hline 4 & 4 & 6 & 4 & 5 & 6 & 4 & 6 & 7 & 5 & 4 & 5 & 3 & 1 & 2 & 3 & 3 & 3 \\
\hline 5 & 8 & 9 & 7 & 5 & 8 & 7 & 6 & 8 & 7 & 4 & 3 & 6 & 7 & 8 & 6 & 6 & 5 \\
\hline
\end{tabular}

Note. Data are from German Socio-Economic Panel Study-2000 (Waves 1984-2000) [Data-file], by the German Institute for Economic Research (DIW), 2002, Berlin, Germany. Copyright 2002 by the German Institute for Economic Research. Reprinted with permission.

stable baseline hypothesis. The mean absolute value of the baseline change was 2.2 scale units for those who experienced a significant change in either direction, whereas the mean absolute value of the baseline change was 0.8 for those who did not experience a significant change. Thus, almost a quarter of participants changed significantly, and those who did so changed substantially. Those who did not change significantly nevertheless changed close to a scale unit in average LS from the beginning to end of the study. The average amount of change from 1 year to the next year was 1.5 scale units, indicating the average amount of change due to recent events and to measurement error in year-to-year measures of LS.

Is the variability of a person's scores related to his or her mean level of LS? We found a significant correlation between the mean of the 17 LS scores and the standard deviations of these judgments $(r=-.47, p<.05)$. The more change in a respondent's satisfaction judgments, the less satisfied she or he was. To ensure that this correlation was not due to ceiling effects (because the majority of participants were in the upper half of the scale), we calculated this correlation again using only participants with an average LS judgment less than the midpoint on the scale. The correlation in the reduced sample remained significant $(r=-.26, n=175, p<.05)$ and in the same direction, despite the restriction of range.

\section{Comparison of Temporal Stability of LS With Other Variables}

If stability in LS is partly due to factors such as life circumstances that tend to change slowly over time, we would expect that the size of correlations between judgments would decrease as the number of years between measurements increases. We computed correlations between all time periods, and we present descriptive statistics on these correlations at the top of Table 2. We then averaged the correlations with the same number of years between measurements. Thus, a single coefficient describes a 16-year span, a mean of 7 coefficients describes a 10-year span, and a mean of 16 coefficients describes a 1-year span. The trend lines in Figure 1 portray the decreasing size of mean correlation coefficients as the number of years between measurements increases. We then conducted a regression analysis predicting the correlation coefficient by the length of span between the two measurements, and because of the concave nature of the curve in Figure 1, we included a squared standardized span predictor. The results of the regression analysis are presented at the bottom of Table 2 .

To compare LS with other variables, we computed correlations between the income reports over time and once again calculated descriptive statistics, averaged correlation coefficients, and conducted a regression analysis on these coefficients. The sample size for the income correlations was smaller $(n=2,771)$ because of listwise deletion of cases with missing data. Figure 1 portrays a similar decline in mean correlation coefficients for income as for LS.

Roberts and Del Vecchio (2000) examined the rank-order consistency, the aspect of stability that we are portraying in Figure 1, of personality traits from childhood to old age. They compiled 3,217 test-retest correlation coefficients from 152 longitudinal studies that included a total sample size of 50,207 participants. Only dispositional variables that were assumed to be consistent, 
Table 2

Descriptive Statistics and Regression Coefficients Using Sets of Correlation Coefficients as Raw Data

\begin{tabular}{|c|c|c|c|c|c|c|c|}
\hline \multirow[b]{2}{*}{ Measure } & \multirow{2}{*}{$\begin{array}{c}\text { Life } \\
\text { satisfaction }\end{array}$} & \multirow[b]{2}{*}{ Income } & \multirow[b]{2}{*}{ Height } & \multirow[b]{2}{*}{ Body mass index } & \multirow[b]{2}{*}{ Weight } & \multicolumn{2}{|c|}{ Blood pressure } \\
\hline & & & & & & Systolic & Diastolic \\
\hline \multicolumn{8}{|l|}{ Descriptive statistics } \\
\hline$n$ & 136 & 136 & 153 & 153 & 276 & 276 & 276 \\
\hline Minimum & 0.239 & 0.207 & 0.910 & 0.514 & 0.661 & 0.164 & 0.101 \\
\hline Maximum & 0.627 & 0.880 & 0.990 & 0.952 & 0.969 & 0.838 & 0.766 \\
\hline$M$ & 0.420 & 0.471 & 0.962 & 0.785 & 0.845 & 0.465 & 0.410 \\
\hline$S D$ & 0.096 & 0.193 & 0.016 & 0.104 & 0.079 & 0.171 & 0.171 \\
\hline \multicolumn{8}{|c|}{$\begin{array}{l}\text { Regression on years between } \\
\text { measurements }\end{array}$} \\
\hline$R^{2}$ & 0.761 & 0.482 & 0.094 & 0.952 & 0.985 & 0.704 & 0.867 \\
\hline Intercept & 0.546 & 0.675 & 0.969 & 0.927 & 0.962 & 0.674 & 0.643 \\
\hline$B$ years & -0.023 & -0.036 & -0.001 & -0.010 & -0.007 & -0.014 & -0.016 \\
\hline$S E B$ years & 0.001 & 0.004 & 0.000 & 0.000 & 0.000 & 0.001 & 0.000 \\
\hline$\beta$ years & -0.934 & -0.732 & -0.339 & -1.017 & -1.034 & -0.904 & -1.005 \\
\hline$B Z$ years $^{2}$ & 0.012 & 0.014 & 0.002 & 0.009 & 0.006 & 0.023 & 0.027 \\
\hline$S E B Z$ years $^{2}$ & 0.004 & 0.012 & 0.001 & 0.002 & 0.001 & 0.005 & 0.004 \\
\hline$\beta Z$ years $^{2}$ & 0.148 & 0.088 & 0.109 & 0.104 & 0.092 & 0.161 & 0.183 \\
\hline
\end{tabular}

enduring, and cross-situational were included. Measures of attitudes, values, self-esteem, affect, mood, intelligence, cognitive functioning, and validity scales were not included. Most of the personality measures were subsumed by the Big Five taxonomy of personality traits. In addition, femininity-masculinity and Type A dimensions were included. In a small portion of their meta-analysis (p. 16), they held age constant at 20 years and estimated average trait consistency at 1-year, 5-year, 10-year, 20-year, and 40-year

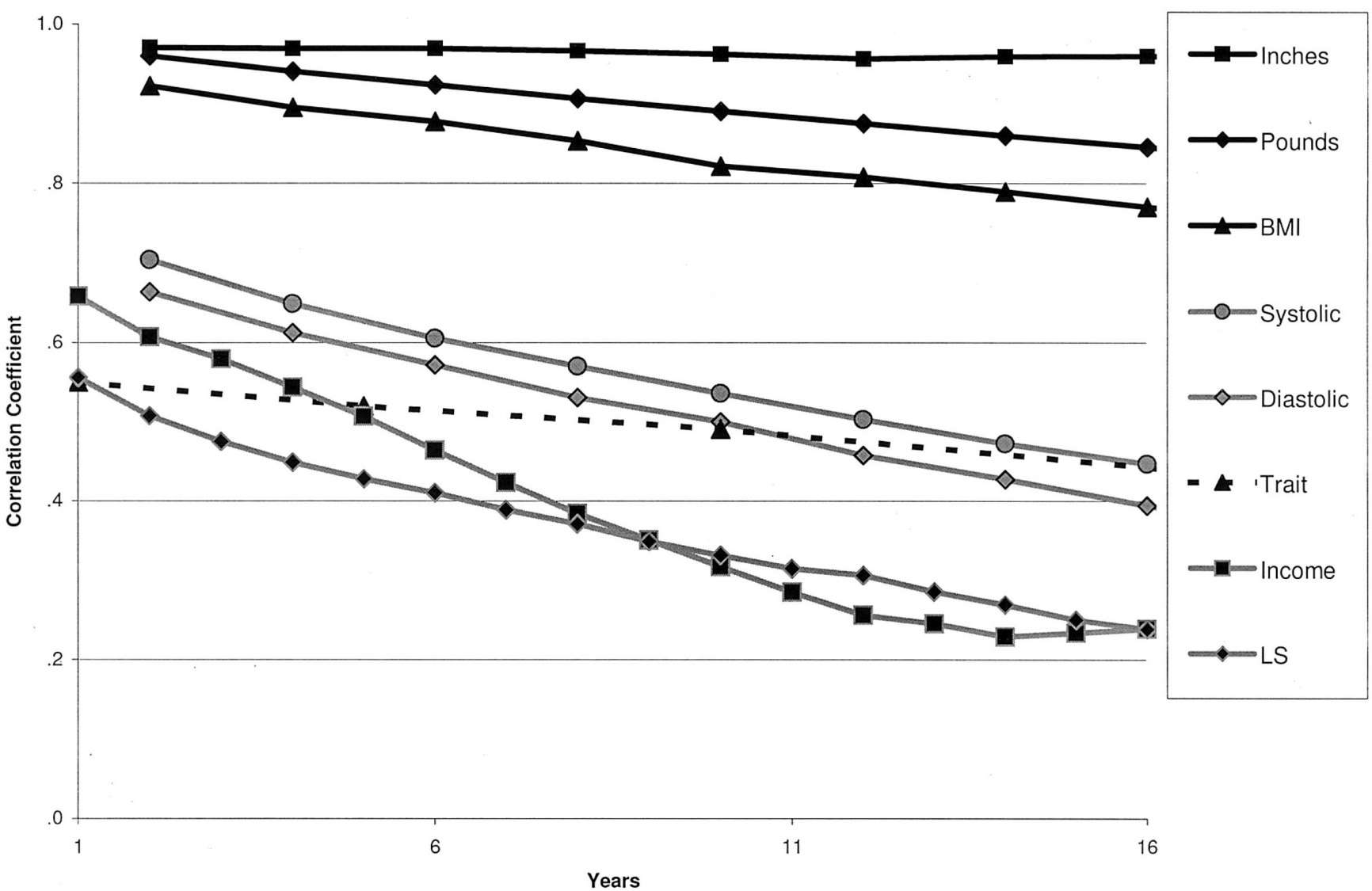

Figure 1. Comparison of stability of psychological, biological, and financial individual differences. BMI $=$ body mass index; LS = life satisfaction. 
time intervals. We incorporated the relevant coefficients into our Figure 1 . The comparison of personality stability with LS stability is important because the other two dimensions of SWB, positive affect and negative affect, are strongly related to the personality dimensions of Extraversion and Neuroticism.

\section{Stability of Biological Measurements Compared With Stability of $L S$}

An analysis of the stability of some biological measurements, like weight, in the same metric that is used to measure stability of LS would provide a meaningful comparison because the baseline conception of LS is based, in part, on the baseline conception of weight. To provide comparisons of the consistency of some biological variables, we used height, weight, and blood pressure data from the Framingham Heart Study (Dawber, Meadors, \& Moore, 1951). Data are reported on 2,336 men and 2,873 women, although the correlations are based on fewer participants because of subject mortality. Weight and blood pressure were measured biannually. Blood pressure was measured at least twice, usually three times, and up to five times per biannual visit. All blood pressure data were averaged to create a single systolic and diastolic measure per biannual visit. Height was measured infrequently at the beginning of the study but biannually in the second half. When both height and weight data were available, the body mass index was calculated. For each type of variable, correlations among variables measured at different times were calculated, descriptive statistics were computed, correlations having the same time interval between measurements were averaged, and a regression analysis was run. The results are plotted on Figure 1 and presented in Table 2.

A visual inspection of the blood pressure correlation matrices, both systolic and diastolic, showed that blood pressure stability depended both on the amount of time between measurements and the average age of the sample. For example, the 2-year systolic stability correlations started at .84 at the beginning of the study when the sample was relatively young and dropped to .54 by the end of the study when the sample was relatively old. The 2-year diastolic stability correlations started at .77 when the sample was young and dropped to .56 when the sample was old. We believe this was due to an increase in importance of the use (or nonuse) of blood-pressure-altering medication and a corresponding decrease in the importance of genetic factors as the Framingham sample aged. Of course, it may simply be the case that blood pressure becomes more variable as one ages. We used the average of all available data to plot the lines in Figure 1.

As can be seen, the physical indicators show much higher stability in adulthood than the other characteristics. It could be argued that their greater stability is in part due to the fact that they are measured more accurately in the first place. Therefore, we computed a disattenuated regression line for LS on the basis of how a similar item performs in the Satisfaction With Life Scale (Diener, Emmons, Larsen, \& Griffin, 1985). In other words, we disattenuated the regression line for measurement error on the basis of the intercorrelation of satisfaction items in other samples. This disattenuated line estimates how well the single LS item would perform if it were error free. The disattenuated line starts at .79 , and is .34 after 16 years, again showing only moderate stability in LS after many years. The correlation of the mean average of the first 5 and last 5 years was $r(3,606)=.51, p<.001$, showing a moderate level of long-term mean average level LS stability.

\section{Discussion}

Our analyses indicate that over long periods of time there is modest stability in LS and that some individuals do change significantly and substantially in LS. Thus, there appears to be a "soft baseline" for LS, with people fluctuating around a stable set point that nonetheless does move for about a quarter of the population. Almost $9 \%$ of the respondents changed 3 or more scale units from the first 5-year average to the final 5-year average. Thus, almost $10 \%$ of the sample showed a change in mean-level satisfaction equal to or greater than 3 points on the 10-point scale, a large change for 5-year averages. Therefore, LS can and does change for some people, even in the face of significant stabilizing factors such as heritable disposition. In this study, we could not analyze the set-point stability for the experience of pleasant and unpleasant emotions, which might be more stable than LS, because these constructs were not included in the GSOEP. It might be that the hedonic treadmill and personality-based set point are more pronounced in measures of moods and emotions, but our data do reveal that some individuals show substantial long-term changes in LS.

Why do our conclusions about the stability of well-being depart from those of Lykken and Tellegen (1996)? We studied LS stability, whereas their idea of a stable set point was primarily focused on people's moods and emotions. It might be that longterm emotional levels are more dependent on inheritance than is a satisfaction judgment, which after all is a judgment made at the time of the survey, and that can be influenced not only by emotions but also by shifting evaluative standards and information that is salient at the moment. Another important fact is that heritable influences can show phenotypic changes over time, with some people increasing and others decreasing on a characteristic because of genetics. Thus, the twins in the Lykken and Tellegen study might have been changing in the same direction over time, but in our study we have no way of knowing whether some of the change we uncovered was due to the influence of heritability. Finally, it should be noted that in the Lykken and Tellegen twin study, there was substantial variance in well-being that was not accounted for by genetics. Although about $80 \%$ of the long-term stability in well-being could be attributed to heritability, long-term stability was only about a quarter of the total variance in well-being scores. Thus, the results presented here appear to be compatible with the idea that there is some stability in well-being that is due to genetics - in fact, we found that about $75 \%$ of individuals did not change significantly in 5-year average well-being even over a period of many years.

Clinical psychologists and others who desire to intervene to improve individuals and society should be encouraged by our findings - the set point of LS can and does change for some individuals. Nevertheless, the majority of individuals do show long-term stability in LS, which is consistent with the idea that a person's inherited temperament exerts an influence on their SWB. Finally, even more stable individuals frequently show changes in LS from one year to the next, which is consistent with the idea that momentary situational factors can influence reports of SWB. Integrating past findings, our results indicate that there are short- 
term, intermediate-term, and long-term influences on SWB. Thus, our results are compatible with the idea of a "soft set point," in which the baseline is not completely fixed because it does change significantly for some individuals.

What part does measurement reliability play in our findings? That is, might LS have been more stable if we had disattenuated the measure because of its unreliability? We approached this question in several ways, and not unexpectedly, stability did rise. Although 1-year reliabilities sometimes rose to levels in the .7 to .8 range, the corrected stabilities after 16 years remained moderate. It is also important to note that the significant change in baseline we found for $24 \%$ of individuals was not due to unreliability of the measures, because significant change was defined in reference to the within-period variability in the measure. That is, the change that occurred was significant when considering the change that might be expected on the basis of the year-to-year changes within the two 5-year time periods.

Why do the stability estimates of all variables that we examined decrease in a regular way over time? In a causal universe in which variables are caused in a systematic way by other preceding factors, it is almost inevitable that the value of a variable will be related to nearby values of that variable more than to distant values, because the conditions causing nearby values are more likely to be similar. Change takes time, and changes normally accumulate over time because of the complex nature of most dynamic systems. The world of today is more likely to be similar to the world of tomorrow than it is to be similar to the world of 100 years from now. Because many of the same causal variables will be in place tomorrow that are in place today, the world is likely to be quite similar tomorrow. Inputs that change an entire system, such as the earth being hit by a huge asteroid, tend to be rare events; otherwise, the systems we are studying would not exist as they do. Furthermore, when a cataclysmic event changes a system, those changes are likely to persist over time (e.g., think of death). The major exception to variables not showing the pattern we found is when there is a cyclical trend in which a particular pattern repeats over time-for example, the four seasons, where the weather 6 months from now is likely to be more different from today than the weather 1 year from now. Although cycles in LS are possible, no long-term cycles have yet been identified. Thus, it is not surprising that LS follows the same pattern of stability as the other variables we examined.

One can imagine specific instances when people will be more stable in the long run than over a shorter time period. For example, if one measured a person's vital signs before a serious illness, they would likely show greater stability a year later after a full recovery than they would after a few days. In this case, we have thought of an instance where the measurements are taken across an important transition event that tends to reverse itself. However, if one takes repeated measurements over time, it is likely that important transition events will increase in likelihood over greater time periods and that people's recovery to former levels will occur only when one specifically seeks people who encounter certain types of reversible events such as short-term illness. When one examines people in the aggregate, such reversible events are likely to influence only a small number of individuals compared with the number of events that produce changes in a specific direction. Furthermore, nonreversible causal events are likely to accumulate as time moves forward.
Our finding that more stable individuals in terms of LS also have a higher mean level of LS is consistent with earlier findings (e.g., Eid \& Diener, 1999). Why might instability be accompanied by lower average LS? For one thing, average levels of LS are above the midpoint of the scale (see also Diener \& Diener, 1996), and people who are high on LS have less room to move around because of ceiling effects, whereas people low in LS are primarily concentrated in the middle of the range where there is more room for movement. However, we suspect that this statistical fact is not solely responsible for the relation between mean levels and variability of well-being. Hepburn and Eysenck (1989) found that people high in neuroticism are more unstable in their moods, and high neuroticism is associated with low LS (Vitterso \& Nilsen, 2002). Furthermore, people with more stable lives have more predictable lives, and this might offer a sense of security and control. Finally, it is likely that people who have more stable life circumstances also have better living conditions in the long term. An ideal set of circumstances is unlikely to include events that can move LS first up, then down, and then back up. For instance, remaining married or single are both likely to be more satisfying than being married, then widowed, then remarried, and then divorced. Because of adaptation, being in one state for a long time (e.g., unemployed) might have less impact than moving in and out of that state (e.g., a cycle of employment and being laid off). Certainly, the issue of why instability is associated with low mean levels of well-being is an intriguing topic for future research. At a practical level, our findings suggest that having either stable life circumstances or a less reactive temperament increases the likelihood of long-term satisfaction.

It is interesting to note that LS is substantially lower in stability than the personality traits considered by Roberts and Del Vecchio (2000). Because psychologists as well as lay people have intuitive notions about the stability of physical characteristics and personality traits, these variables give us a feel for the stability of LS. The finding that personality traits are more stable than LS suggests that although temperament might influence both variables, bottom-up environmental factors, which are more unstable than genetic influences, exert a larger influence on LS than on personality traits. Because LS is influenced by personality and yet is less stable than personality, the influence of changeable environmental factors appears to substantially influence well-being, again pointing to the fact that the set point for LS is not set in concrete. In the debate about whether well-being is a trait or state, our findings alongside Roberts and Del Vecchio's results suggest that LS is somewhat stable but less so than the average personality trait.

It is also interesting to compare LS stability with that of other measures. Height, not surprisingly, is extremely stable. Weight, body mass index, systolic and diastolic blood pressure, and personality traits are all fairly stable. Only income matches the moderate stability levels of LS, which is a helpful comparison, because we have intuitive notions about income. We know that relative income has some stability because of education, one's profession, and other factors, but we also know that some people lose their jobs and income, whereas others find lucrative new opportunities. Like relative income, LS appears to be relatively stable (but with small year-to-year perturbations) for most individuals most of the time, but it does change substantially in some cases.

To return to the original question, is there a stable set point for LS? The set-point idea has been criticized even for weight, because 
factors such as exercise and exposure to high-calorie foods can alter people's baselines. Nevertheless, the idea of set point captures the truth that some individuals, at least within the typical diet of their society, have an easy time remaining thin, whereas others must exert enormous effort to avoid obesity. As with weight, some people seem to remain relatively satisfied with their lives over long periods of time, and others seem to have low or fluctuating LS even over a period as long as 17 years. We suspect that both inborn temperament and life circumstances are at work, just as they are for weight. However, because LS is a judgment that is created by the individual and can take different information into account on different occasions, it might follow a set-point model less than would average levels of positive and negative affect, which depend more heavily on genes and temperament.

Why might people's set-point levels of LS change over time? The findings on marital status changes by Lucas et al. (2003) suggest that factors such as widowhood can create long-lasting downward pressures on LS. Although Lucas et al. did not find that on average marriage permanently increased LS, they did find that this was true for some individuals. Some respondents increased their set point after marriage, even though they adapted to the event over time to some degree, whereas other people decreased in their long-term LS after marriage. Although statistics based on entire populations might not show long-lasting mean changes after important life events, this in some cases seems to be due to the fact that some people change upward and others change downward in reaction to the same event. Another event that appears capable of moving people's LS downward is unemployment (Lucas et al., 2004).

Mapping the personality and life circumstance changes that can alter a person's baseline level of well-being is a top priority for the science of well-being. The stability of well-being is often high and is substantially influenced by one's inherited predispositions. The challenge is to discover what life changes produce only short-term changes in well-being and are followed by a return to the set point and what types of events cause long-term changes in well-being.

\section{References}

Brickman, P., \& Campbell, D. T. (1971). Hedonic relativism and planning the good society. In M. H. Apley (Ed.), Adaptation-level theory (pp. 287-305). New York: Academic Press.

Brickman, P., Coates, D., \& Janoff-Bulman, R. (1978). Lottery winners and accident victims: Is happiness relative? Journal of Personality and Social Psychology, 36, 917-927.

Dawber, T. R., Meadors, G. F., \& Moore, F. E. J. (1951). Epidemiological approaches to heart disease: The Framingham Study. American Journal of Public Health, 41, 279-286.

Diener, E., \& Diener, C. (1996). Most people are happy. Psychological Science, 7, 181-185.

Diener, E., Emmons, R. A., Larsen, R. J., \& Griffin, S. (1985). The Satisfaction With Life Scale. Journal of Personality Assessment, 49, 71-75.

Eid, M., \& Diener, E. (1999). Intraindividual variability in affect: Reli- ability, validity, and personality correlates. Journal of Personality and Social Psychology, 76, 662-676.

Eid, M., \& Diener, E. (2004). Global judgments of subjective well-being: Situational variability and long-term stability. Social Indicators Research, 65, 245-277.

German Institute for Economic Research (DIW). German Socio-Economic Panel Study-2002 (Waves 1984-2000) [Data file]. Berlin, Germany: Author.

Haisken-De New, J. P., \& Frick, R. (1998). Desktop companion to the German Socio-Economic Panel Study (GSEOP). Berlin, Germany: German Institute for Economic Research.

Headey, B., \& Wearing, A. (1992). Understanding happiness: A theory of subjective well-being. Melbourne, Victoria, Australia: Longman Cheshire.

Hepburn, L., \& Eysenck, M. W. (1989). Personality, average mood and mood variability. Personality and Individual Differences, 10, 975-983.

Inglehart, R., \& Klingemann, H. -D. (2000). Genes, culture, democracy, and happiness. In E. Diener \& E. M. Suh (Eds.), Culture and subjective well-being (pp. 165-183). Cambridge, MA: MIT Press.

Kahneman, D., Diener, E., \& Schwarz, N. (1999). Well-being: The foundations of hedonic psychology. New York: Russell Sage Foundation.

Lucas, R. E., Clark, A. E., Georgellis, Y., \& Diener, E. (2003). Reexamining adaptation and the set point model of happiness: Reactions to changes in marital status. Journal of Personality and Social Psychology, $84,527-539$.

Lucas, R. E., Clark, A. E., Georgellis, Y., \& Diener, E. (2004). Unemployment alters the set-point for life satisfaction. Psychological Science, $15,8-13$

Lykken, D. (1999). Happiness: What studies on twins show us about nature, nurture, and the happiness set-point. New York: Golden Books.

Lykken, D. T., \& Tellegen, A. (1996). Happiness is a stochastic phenomenon. Psychological Science, 7, 186-189.

Myers, D. G. (1992). The pursuit of happiness. New York: William Morrow.

Roberts, B. W., \& Del Vecchio, W. F. (2000). The rank-order consistency of personality from childhood to old age: A quantitative review of longitudinal studies. Psychological Bulletin, 126, 3-25.

Schimmack, U., Diener, E., \& Oishi, S. (2002). Life-satisfaction is a momentary judgment and a stable personality characteristic: The use of chronically accessible and stable sources. Journal of Personality, 70, 345-384.

Schwarz, N., \& Strack, F. (1999). Reports of subjective well-being: Judgmental processes and their methodological implications. In D. Kahneman, E. Diener, \& N. Schwarz (Eds.), Well-being: The foundations of hedonic psychology (pp. 61-85). New York: Russell Sage Foundation.

Tellegen, A., Lykken, D. T., Bouchard, T. J., Wilcox, K. J., Segal, N. L., \& Rich, S. (1988). Personality similarity in twins reared apart and together. Journal of Personality and Social Psychology, 54, 1031-1039.

Vitterso, J., \& Nilsen, F. (2002). The conceptual and relational structure of subjective well-being, neuroticism, and extraversion: Once again, neuroticism is the important predictor of happiness. Social Indicators Research, 57, 89-118.

Received January 29, 2004

Revision received July 16, 2004

Accepted July 19, 2004 